ARTICLE

DOI: $10.1038 /$ s41467-017-02498-

\title{
One-step fermentative production of aromatic polyesters from glucose by metabolically engineered Escherichia coli strains
}

\author{
Jung Eun Yang ${ }^{1}$, Si Jae Park ${ }^{2}$, Won Jun Kim¹, Hyeong Jun Kim³ ${ }^{3}$ Bumjoon J. Kim³ ${ }^{3}$ Hyuk Lee ${ }^{4}$,
} Jihoon Shin $^{5} \&$ Sang Yup Lee (1) ${ }^{1,6,7}$

Aromatic polyesters are widely used plastics currently produced from petroleum. Here we engineer Escherichia coli strains for the production of aromatic polyesters from glucose by one-step fermentation. When the Clostridium difficile isocaprenoyl-CoA:2-hydroxyisocaproate CoA-transferase (HadA) and evolved polyhydroxyalkanoate (PHA) synthase genes are overexpressed in a D-phenyllactate-producing strain, poly(52.3 mol\% 3-hydroxybutyrate (3HB)-co-47.7 mol\% D-phenyllactate) can be produced from glucose and sodium $3 \mathrm{HB}$. Also, various poly(3HB-Co-D-phenyllactate) polymers having 11.0, 15.8, 20.0, 70.8, and 84.5 mol\% of $D$-phenyllactate are produced from glucose as a sole carbon source by additional expression of Ralstonia eutropha $\beta$-ketothiolase ( $p h a A$ ) and reductase ( $p h a B)$ genes. Fed-batch culture of this engineered strain produces $13.9 \mathrm{gl}^{-1}$ of poly $61.9 \mathrm{~mol} \% 3 \mathrm{HB}-\mathrm{co}-38.1 \mathrm{~mol} \% \mathrm{D}-$ phenyllactate). Furthermore, different aromatic polyesters containing D-mandelate and D-3hydroxy-3-phenylpropionate are produced from glucose when feeding the corresponding monomers. The engineered bacterial system will be useful for one-step fermentative production of aromatic polyesters from renewable resources.

\footnotetext{
${ }^{1}$ Metabolic and Biomolecular Engineering National Research Laboratory, Department of Chemical and Biomolecular Engineering (BK21 Plus Program), Center for Systems and Synthetic Biotechnology, Institute for the BioCentury, Korea Advanced Institute of Science and Technology (KAIST), Daejeon 34141, Republic of Korea. ${ }^{2}$ Division of Chemical Engineering and Materials Science, Ewha Womans University, Seoul 03760, Republic of Korea. ${ }^{3}$ Polymer and Nano Electronics Laboratory, Department of Chemical and Biomolecular Engineering (BK21 Plus Program), Institute for the BioCentury, KAIST, Daejeon 34141, Republic of Korea. ${ }^{4}$ Division of Drug Discovery Research, Korea Research Institute of Chemical Technology, Daejeon 34114, Republic of Korea. ${ }^{5}$ Center for Bio-based Chemistry, Green Chemistry \& Engineering Division, Korea Research Institute of Chemical Technology, Daejeon 34114, Republic of Korea. ${ }^{6}$ BioProcess Engineering Research Center, KAIST, Daejeon 34141, Republic of Korea. ${ }^{7}$ Biolnformatics Research Center, KAIST, Daejeon 34141 , Republic of Korea. Jung Eun Yang and Si Jae Park contributed equally to this work. Correspondence and requests for materials should be addressed to S.Y.L. (email: leesy@kaist.ac.kr)
} 
A romatic polyesters are widely used indispensable plastics currently produced from petroleum ${ }^{1}$. Microbial fermentative production of polymers from renewable resources has received much attention to substitute petroleum-based polymers and help solving environmental problems. Thus, there has been much interest in fermentative production of aromatic polyesters from renewable non-food biomass, but without any success. Polyhydroxyalkanoates (PHAs) comprising various hydroxycarboxylic acids are natural biodegradable microbial polyesters, which are accumulated by numerous microorganisms under nutrient limited conditions in the presence of excess carbon sources 2,3 . The material properties of PHAs can be modulated by changing the monomer types and compositions. Over the last three decades, numerous metabolic engineering studies have been performed to produce PHAs containing specific monomers having different monomer compositions. Various kinds of monomers such as 3-hydroxypropionate, 3-hydroxybutyrate (3HB), 3-hydroxyvalerate, 4-hydroxybutyrate, 5-hydroxyvalerate, and 6-hydroxyhexanoate, and medium-chain length 3-hydroxyalkanoates have been shown to be incorporated into PHAs, either as homo- or co-polymers, giving diverse material properties $^{2,4-7}$.

More recently, one-step fermentative production of nonnatural polyesters, poly(D-lactate), poly(lactate-co-glycolate), and other D-lactate-containing PHAs by metabolically engineered bacteria have been reported ${ }^{7-13}$. In these studies, an evolved propionate CoA-transferase (Pct) and an evolved PHA synthase (PhaC) were expressed in Escherichia coli; engineered Clostridium propionicum Pct $_{c p}$ converts 2-hydroxyacids to corresponding 2-hydroxyacyl-CoAs using acetyl-CoA as a CoA-donor and then engineered Pseudomonas sp. MBEL 6-19 PHA synthase $\left(\mathrm{PhaC}_{P_{\mathrm{s} 6-19}}\right)$ polymerizes these 2-hydroxyacyl-CoAs into polyesters containing corresponding monomers ${ }^{9-11}$. It has previously been reported that some bacteria such as Pseudomonas oleovorans and Pseudomonas putida strains can synthesize aromatic polyesters when grown in the culture medium containing $n$-phenylalkanoic acid as a direct precursor of aromatic monomer ${ }^{14-16}$. These aromatic polyesters have only been produced by feeding the cells with corresponding aromatic monomers as substrates and have not been produced by direct fermentation from renewable feedstock carbohydrates such as glucose. Furthermore, those mentioned above contain aromatic groups far away from the main polymer carbon chain ${ }^{14-16}$ and thus polymer properties were found to be much different from petroleum-derived aromatic polymers, which often contain aromatic rings close to the main polymer chain, such as poly(ethylene terephthalate) (PET) and polystyrene. Such observations led us to develop metabolically engineered E. coli strains for one-step fermentative production of aromatic polyesters from glucose.

The following strategies were employed in this study. First, CoA-transferases that can efficiently activate phenylalkanoates into their corresponding CoA derivatives were discovered. Second, cells were metabolically engineered to produce phenylalkanoates from glucose. Third, the engineered phenylalkanoates overproducing $E$. coli strains were employed for in vivo production of aromatic polyesters by expressing engineered PHA synthase and CoA-transferases. Fourth, strains were further engineered to produce aromatic polyesters directly from glucose. Fifth, the enzyme expression levels were modulated to produce various aromatic polyesters having different monomer fractions. Finally, as proof-of-concept examples of expanding the range of aromatic polyesters that can be produced by fermentation, aromatic polyesters containing D-mandelate and D-3-hydroxy-3phenylpropionate were produced by feeding the corresponding precursors.

\section{Results}

Validation of the CoA-transferase activity. First, we examined whether Pct can activate phenyllactate and mandelate into phenyllactyl-CoA and mandelyl-CoA, respectively. As the mutant of Pct $_{c p}$ (Pct540) has successfully been employed for in vivo production of polyesters containing 2-hydroxyacids such as glycolate, lactate, 2-hydroxybutyrate, and 2-hydroxyisovalerate, and various hydroxyacids ${ }^{7}$, the substrate spectrum of Pct540 seems to be broad enough with respect to the number of carbon atoms and position of hydroxyl group. Unfortunately, however, Pct540 was found to have no catalytic activities toward phenyllactate and mandelate (Supplementary Fig. 1). Thus, a CoA-transferase capable of activating aromatic compounds into corresponding CoA derivatives had to be identified for aromatic polymer production.

There have been reports describing that Clostridium sporogenes cinnamoyl-CoA:phenyllactate CoA-transferase (FldA) can convert phenyllactate into phenyllactyl-CoA using cinnamoyl$\mathrm{CoA}$ as a CoA-donor ${ }^{17}$. As cinnamoyl-CoA is a non-natural metabolite in E. coli, the possibility of using acetyl-CoA, which is abundant metabolite in the cell, as a CoA-donor was examined for the synthesis of phenyllactyl-CoA by Clostridium botulinum A str. ATCC 3502 FldA (using the synthesized gene), which shared $99.0 \%$ of amino acid sequence identity with that of $C$. sporogenes. However, FldA was found to have no catalytic activity on generating phenyllactyl-CoA using acetyl-CoA as a CoA-donor. A previous study reported that Streptomyces coelicolor 4-coumarate:CoA ligase (4CL) has an important role in phenylpropanoid metabolism via generating cinnamoyl-CoA from cinnamate ${ }^{18}$. Thus, a biosynthetic route was designed and tested in vitro for the synthesis of cinnamoyl-CoA from cinnamate by 4CL (Supplementary Fig. 2 and Supplementary Note 1). A modified $4 \mathrm{CL}^{18}$ was applied to make cinnamoyl-CoA, which can be used as CoA-donor for FldA mediated phenyllactylCoA formation. As expected, phenyllactyl-CoA was successfully synthesized by in vitro sequential reaction of $4 \mathrm{CL}$ and FldA; $4 \mathrm{CL}$ converts cinnamate to cinnamoyl-CoA and then FldA converts phenyllactate into phenyllactyl-CoA (Supplementary Fig. 3). These results suggest that $4 \mathrm{CL}$ and FldA can potentially be used for phenyllactyl-CoA generation and consequently aromatic polyester production. Similarly, 4-hydroxyphenyllactate, another promising aromatic monomer, was also converted to 4-hydroxyphenyllactyl-CoA by in vitro sequential reaction of mutant 4CL and FldA (Supplementary Fig. 3).

In vivo polymerization of aromatic PHA using FldA. In the biosynthesis of non-natural polyesters, selection of a monomerspecific $\mathrm{PhaC}$ variant is crucial for the production of desired polyesters. To examine the performance of various PhaCs on aromatic PHAs production, engineered Pseudomonas sp. MBEL 6-19 PHA synthase $\left(\mathrm{PhaC}_{P \mathrm{~s} 6-19}\right)$ variants (PhaC1202, $\mathrm{PhaC} 1301$, PhaC1310, PhaC1437, and PhaC1439) ${ }^{10}$ were expressed in E. coli XL1-Blue strain together with 3-deoxy-D-arabino-heptulosonate7-phosphate (DAHP) synthase $\left(\mathrm{AroG}^{\mathrm{fbr}}\right)$, phenylalanine ammonia-lyase (PAL), 4CL, FldA, and Pct540 in MR medium (see Methods) containing $20 \mathrm{~g}^{-1}(111 \mathrm{mM})$ of glucose, $1 \mathrm{~g} \mathrm{l}^{-1}$ of D-phenyllactate, and $1 \mathrm{~g} \mathrm{l}^{-1}$ of sodium $3 \mathrm{HB}$. Here, $3 \mathrm{HB}$ was supplied to enhance the production of polymers, because it is converted by Pct540 into 3HB-CoA, a more favorable substrate of $\mathrm{PhaC}$, thus allowing production of PHAs to sufficient amounts for further analysis. The E. coli XL1-Blue strains expressing different PHA synthase variants together with AroG ${ }^{\mathrm{fbr}}, \mathrm{PAL}, 4 \mathrm{CL}$, FldA, and Pct540 were able to produce varying amounts of random copolymers, poly(D-lactate-co-3HB-co-D-phenyllactate), having different monomer compositions (Supplementary Fig. 4a). 


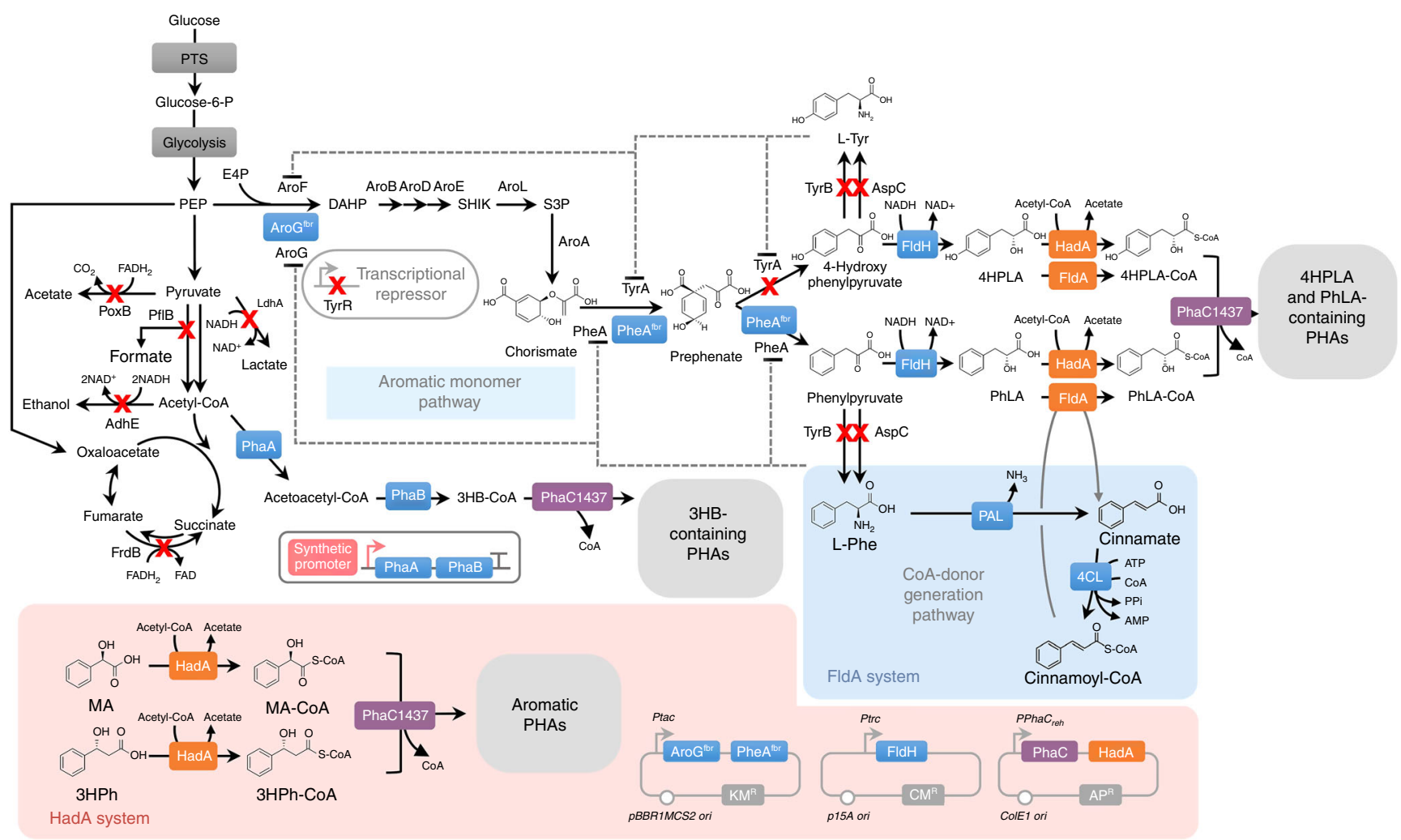

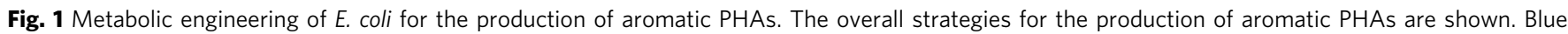
boxes represent heterologous and endogenous enzymes introduced for the construction of metabolic pathways for aromatic PHAs. The orange and purple boxes indicate CoA-transferase and PHA synthase, respectively. The inactivated metabolic pathways are indicated by " $X$ ". Dotted lines indicate feedback inhibition. DAHP, 3-deoxy-D-arabino-heptulosonate-7-phosphate; E4P, erythrose-4-phosphate; 3HB-CoA, 3-hydroxybutyryl-CoA; 3HPh, D-3-hydroxy-3-

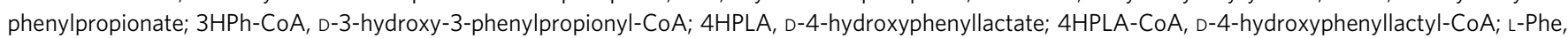
L-phenylalanine; L-Tyr, L-tyrosine; PhLA, D-phenyllactate; MA, D-mandelate; MA-CoA, D-mandelyl-CoA; PhLA-CoA, D-phenyllactatyl-CoA; PEP,

phosphoenolpyruvate; SHIK, shikimate; S3P, shikimate-3-phosphate

Among the PhaC variants, PhaC1437 containing four amino acids substitutions (E130D, S325T, S477G, and Q481K) was found to be the best, which resulted in the production of poly(18.3 mol\% D-lactate-co-76.9 mol\% $\quad 3 \mathrm{HB}-c o-4.8 \mathrm{~mol} \%$ D-phenyllactate) to the polymer content of $7.8 \mathrm{wt} \%$ of dry cell weight (Supplementary Fig. 4a, b).

As externally supplemented D-phenyllactate was successfully incorporated into PHAs, it was aimed to engineer E. coli to generate D-phenyllactate in vivo from glucose directly. Biosynthesis of aromatic compounds starts from DAHP, which is derived from the condensation of phosphoenolpyruvate and erythrose-4phosphate by DAHP synthase. The DAHP is converted into phenylpyruvate and then further converted to D-phenyllactate by D-phenyllactate dehydrogenase (FldH) (Fig. 1). It is well known that the metabolic pathways for the biosynthesis of aromatic compounds are regulated by various inhibitory mechanisms ${ }^{19}$; expression of DAHP synthase encoded by aroG and chorismate mutase/prephenate dehydratase encoded by pheA are inhibited by $\mathrm{L}$-phenylalanine $\mathrm{e}^{20}$. To produce $\mathrm{D}$-phenyllactic acid in vivo, negative feedback inhibition resistant mutants $\mathrm{AroG}^{\mathrm{fbr}}$ [AroG $(\mathrm{D} 146 \mathrm{~N})]$ and $\mathrm{PheA}^{\mathrm{fbr}}[\mathrm{PheA}(\mathrm{T} 326 \mathrm{P})]$ were constructed based on the previous reports ${ }^{21,22}$. The engineered E. coli XL1-Blue strain expressing AroG ${ }^{\mathrm{fbr}}$, PheA ${ }^{\mathrm{fbr}}$, and the C. botulinum A str. ATCC $3502 \mathrm{FldH}$ was able to produce $0.372 \mathrm{gl}^{-1}$ of D-phenyllactic acid from $15.2 \mathrm{gl}^{-1}(84.4 \mathrm{mM})$ of glucose. Moreover, additional overexpression of PAL, 4CL, FldA, Pct540, and $\mathrm{PhaC} 1437$ in this strain resulted in production of poly $(16.8 \mathrm{~mol} \%$ D-lactate-co- 80.8 mol\% $3 \mathrm{HB}-c o-1.6 \mathrm{~mol} \%$ D-phenyllactate-co- 0.8 mol\% D-4-hydroxyphenyllactate) to the polymer content of 12.8 wt $\%$ of dry cell weight in MR medium containing $20 \mathrm{gl}^{-1}$ of glucose and $1 \mathrm{gl}^{-1}$ of sodium 3HB (Supplementary Fig. 5 and Supplementary Table 1). It should be noted that a small amount of D-4-hydroxyphenyllactate was also incorporated into the polymer, which is predictable from in vitro FldA assay (Supplementary Fig. 3); D-4-hydroxyphenyllactyl-CoA was also generated by FldA and polymerized with D-phenyllactyl-CoA.

Identification of 2-hydroxyisocaproate CoA-transferase. Although aromatic PHAs containing D-phenyllactate could be successfully produced, critical problems still exist in using the FldA system, such as rather low aromatic monomer content and narrow monomer spectrum. In vitro enzyme assay results suggested that FldA was able to transfer CoA to phenyllactate and 4-hydroxyphenyllactate, but not to others of our interest, such as mandelate, 2-hydroxy-4-phenylbutyrate, 3-hydroxy-3-phenylpropionate, and 4-hydroxybenzoate (Supplementary Fig. 3). Thus, the Clostridium difficile isocaprenoyl-CoA:2-hydroxyisocaproate CoA-transferase (HadA), which has more than $48 \%$ of amino acid sequence identity to FldA was newly identified based on amino acid sequence similarity analysis (Supplementary Fig. 6).

Interestingly, despite isocaprenoyl-CoA-based 2-hydroxyisocaproate-specific CoA transferring activity of $\mathrm{HadA}^{23}$, we newly discovered that phenyllactate could be converted to phenyllactylCoA using acetyl-CoA derived CoA moiety (Fig. 2 and Supplementary Fig. 7). Moreover, HadA showed catalytic activity on converting mandelate, 4-hydroxymandelate, 4- 
hydroxyphenyllactate, 2-hydroxy-4-phenylbutyrate, 3-hydroxy-3phenylpropionate, and 4-hydroxybenzoate to their corresponding CoA derivatives (Fig. 2 and Supplementary Fig. 7c-k). Thus, HadA has a potential to more efficiently produce diverse aromatic polyesters using acetyl-CoA as a CoA donor.

a

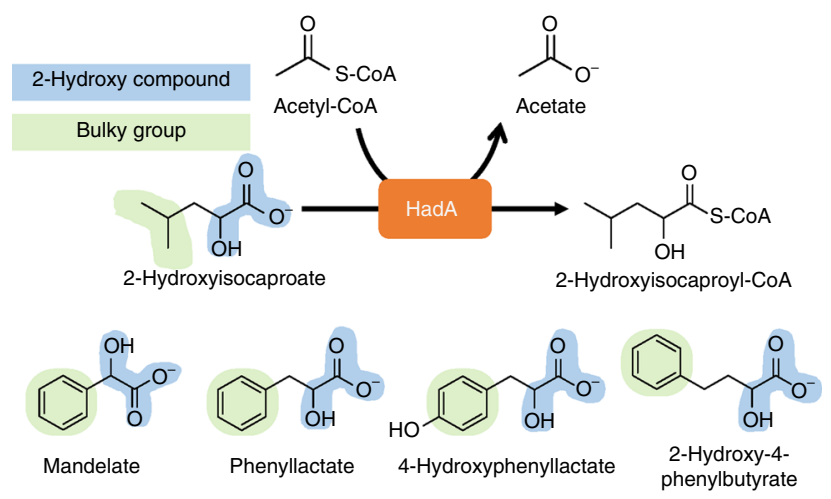

Enhanced carbon flux for D-phenyllactic acid production. For the overproduction of $\mathrm{D}$-phenyllactate, the $t y r R$ gene was deleted to make E. coli XBT strain due to the negative regulation of TyrR on aromatic amino acid biosynthesis. E. coli XBT strain expressing AroG ${ }^{\mathrm{fbr}}$, PheA ${ }^{\mathrm{fbr}}$, and FldH could produce $0.5 \mathrm{gl}^{-1}$ of $\mathrm{D}$ -
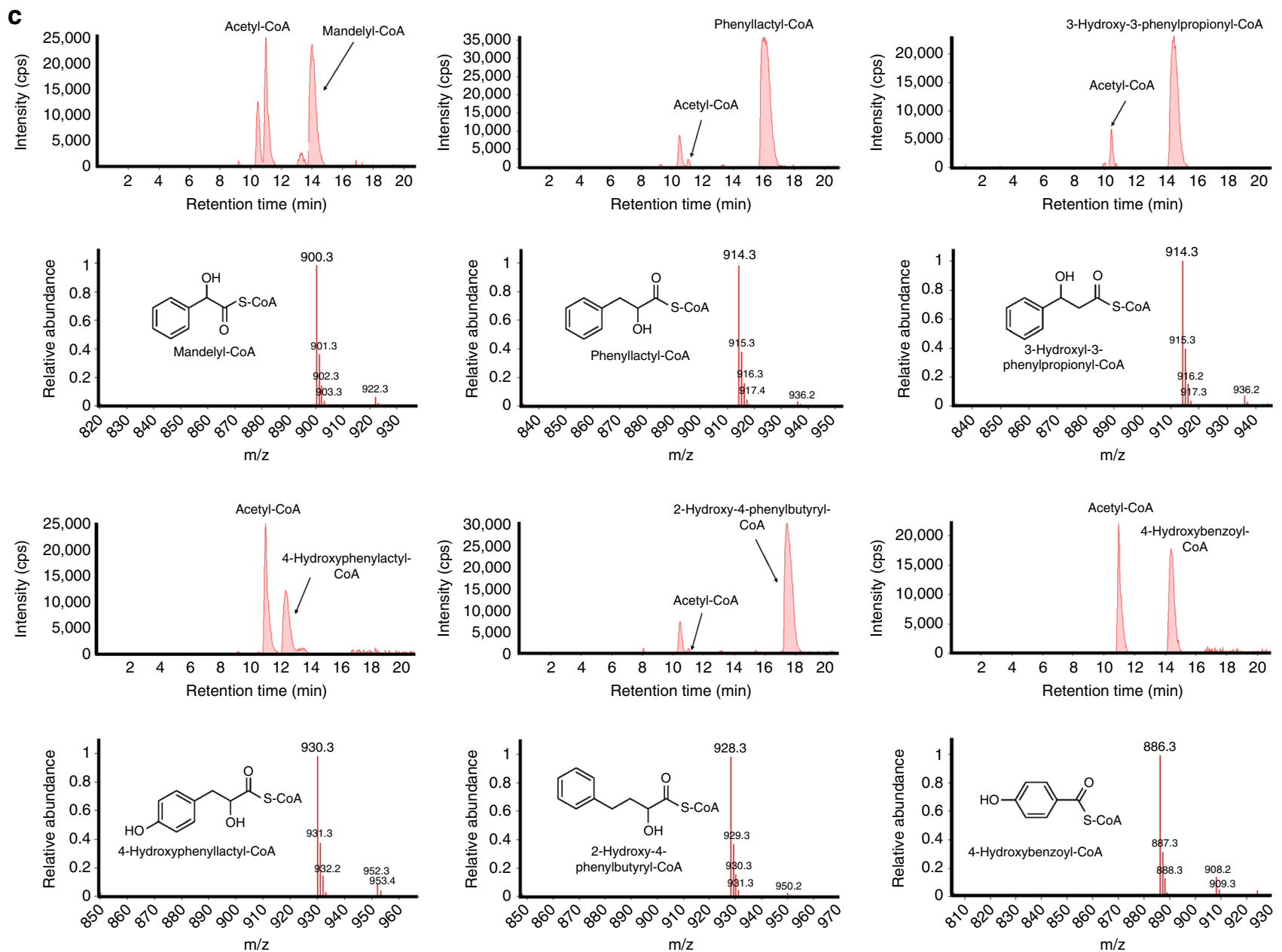

Fig. 2 Activity of HadA on various substrates in vitro. a Schematic representation of reaction used in the assay. $\mathbf{b}$ The specific activities of HadA on various substrates. Data represent mean \pm SD ( $n=3$ technical replicates). c LC-MS analysis of various aromatic acyl-CoAs generated by HadA during the in vitro assay 
a

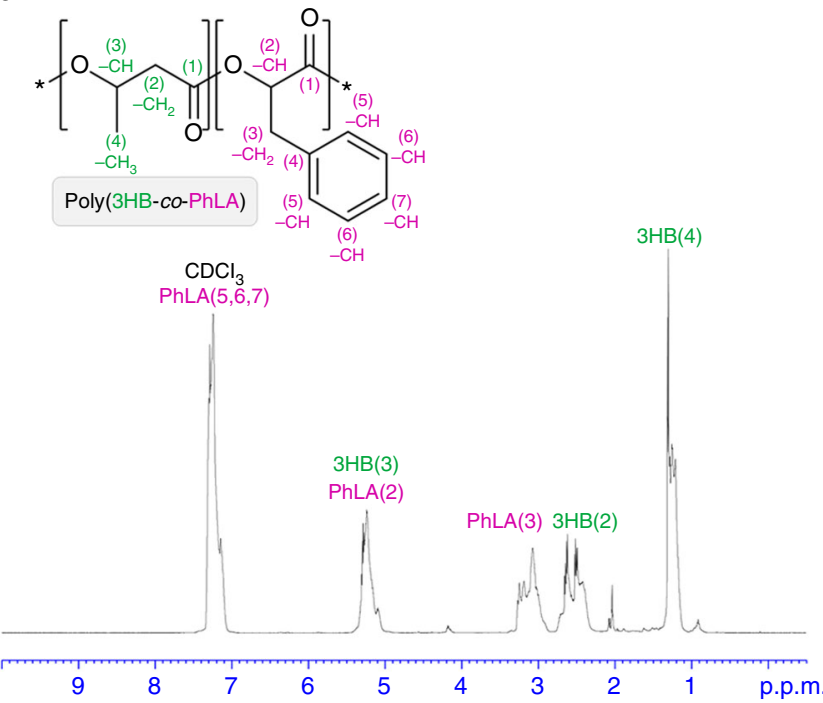

b

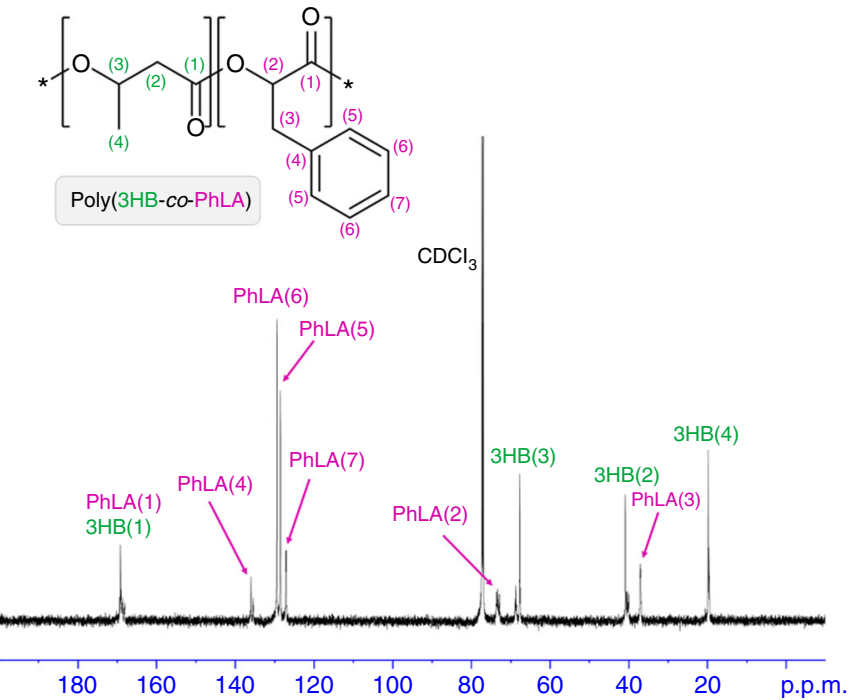

C

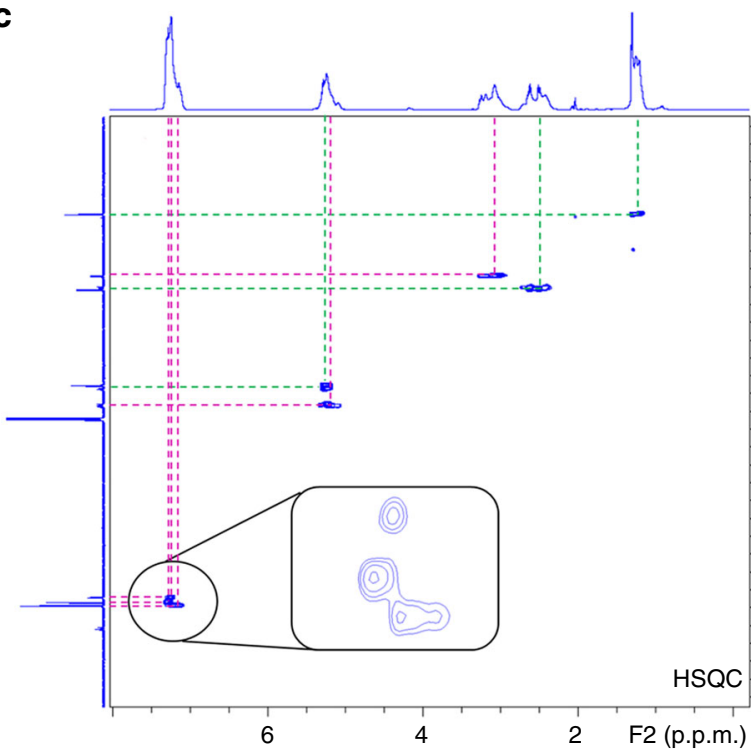

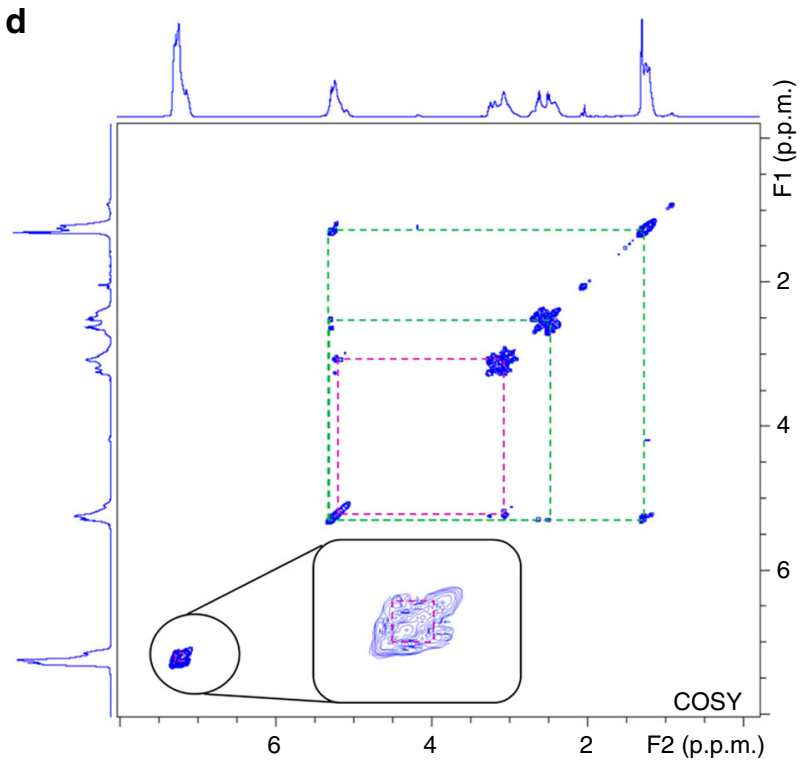

Fig. 3 Characterization of aromatic PHAs produced by metabolically engineered E. coli. a-d NMR spectra of poly(3HB-co-D-phenyllactate) produced in $E$. coli XB201TBAL expressing AroG ${ }^{\mathrm{fbr}}$, PheA ${ }^{\mathrm{fbr}}$, FldH, HadA, and PhaC1437. a ${ }^{1} \mathrm{H}$ NMR. b ${ }^{13} \mathrm{C}$ NMR. c $2 \mathrm{D}{ }^{1} \mathrm{H}-{ }^{13} \mathrm{C}$ HSQC NMR. d $2 \mathrm{D}{ }^{1} \mathrm{H}-{ }^{1} \mathrm{H}$ COSY NMR

phenyllactic acid from $16.4 \mathrm{gl}^{-1}(91 \mathrm{mM})$ of glucose, which is $34.4 \%$ higher than that obtained with E. coli XL1-Blue strain expressing the same genes. In order to remove competing pathways of D-phenyllactate biosynthesis, the poxB (encoding pyruvate oxidase), $p f l B$ (encoding pyruvate formate lyase), adhE (encoding acetaldehyde dehydrogenase/alcohol dehydrogenase), and $f r d B$ (encoding fumarate reductase) genes were deleted in E. coli XBT, to make E. coli XB201T. The E. coli XB201T strain expressing AroG ${ }^{\mathrm{fbr}}$, PheA ${ }^{\mathrm{fbr}}$, and FldH produced $0.55 \mathrm{gl}^{-1}$ of D-phenyllactic acid from $15.7 \mathrm{~g}^{-1}(87 \mathrm{mM})$ of glucose, which is $10 \%$ higher than that obtained with the XBT strain (Supplementary Fig. 8). The tyrB gene encoding tyrosine aminotransferase and $a s p C$ gene encoding aspartate aminotransferase were also deleted, to increase carbon flux toward D-phenyllactic acid based on in silico genome-scale metabolic flux analyses (Supplementary Note 2). The resulting E. coli XB201TBA strain expressing $A \mathrm{AroG}^{\mathrm{fbr}}$, $\mathrm{PheA}^{\mathrm{fbr}}$, and FldH allowed significantly enhanced production of D-phenyllactic acid up to $1.62 \mathrm{~g} \mathrm{l}^{-1}$ from $18.5 \mathrm{~g}^{-1}(102 \mathrm{mM})$ of glucose, which is 4.35 -fold higher than that obtained with the original XL1-Blue strain expressing the same genes. The $\operatorname{ldh} A$ gene was further deleted in XB201TBA to prevent D-lactate formation, to make XB201TBAL strain. The engineered $E$. coli XB201TBAL strain expressing AroG ${ }^{\mathrm{fbr}}$, PheA $^{\mathrm{fbr}}$, FldH, HadA, and PhaC1437 was able to produce poly (52.1 mol\% $3 \mathrm{HB}-\mathrm{co}-47.9 \mathrm{~mol} \% \mathrm{D}$-phenyllactate) to the polymer content of $15.8 \mathrm{wt} \%$ of dry cell weight in MR medium containing $20 \mathrm{~g} \mathrm{l}^{-1}$ of glucose and $1 \mathrm{~g} \mathrm{l}^{-1}$ of sodium $3 \mathrm{HB}$ (Fig. 3). It should be noted that $\mathrm{D}$-4-hydroxyphenyllactate was not incorporated into the polymer anymore through reinforcing the flux toward D-phenyllactate. Moreover, poly $(52.3 \mathrm{~mol} \% 3 \mathrm{HB}-\mathrm{co}-47.7 \mathrm{~mol} \%$ D-phenyllactate) could be produced to a higher polymer content of $24.3 \mathrm{wt} \%$ by fed-batch fermentation (Supplementary Fig. 9).

The importance of HadA in the production of PHAs containing diverse aromatic monomers was also evaluated by supplementation of monomers of interest such as D-mandelate and D-3-hydroxy-3-phenylpropionate. The final engineered E. coli XB201TBAL expressing AroG ${ }^{\mathrm{fbr}}$, $\mathrm{PheA}^{\mathrm{fbr}}$, FldH, HadA, and PhaC1437 was able to produce poly(55.2 mol\% 3HB-co-43.0 mol $\%$ D-phenyllactate-co- $1.8 \mathrm{~mol} \% \mathrm{D}$-mandelate) to the polymer content of $11.6 \mathrm{wt} \%$ of dry cell weight and poly $(33.3 \mathrm{~mol} \%$ 
a

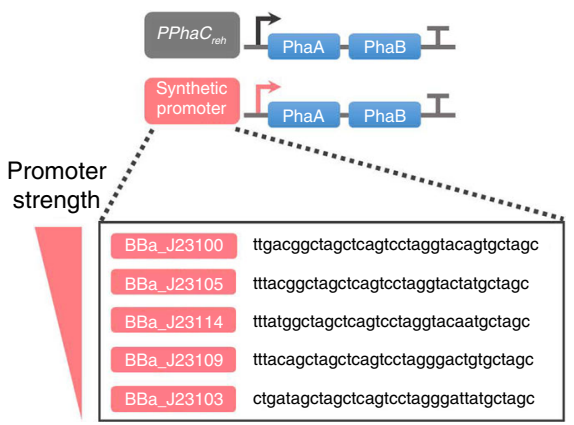

b

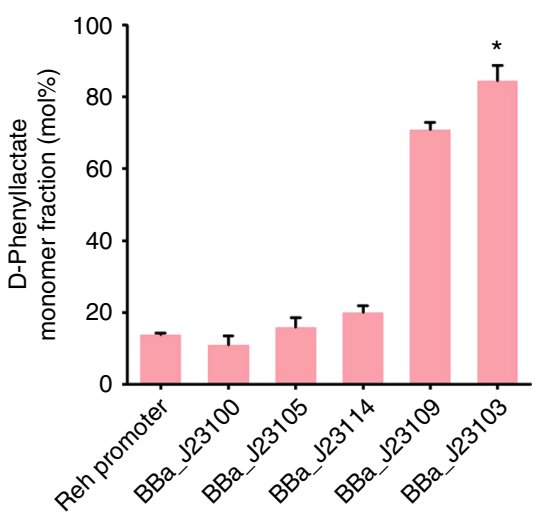

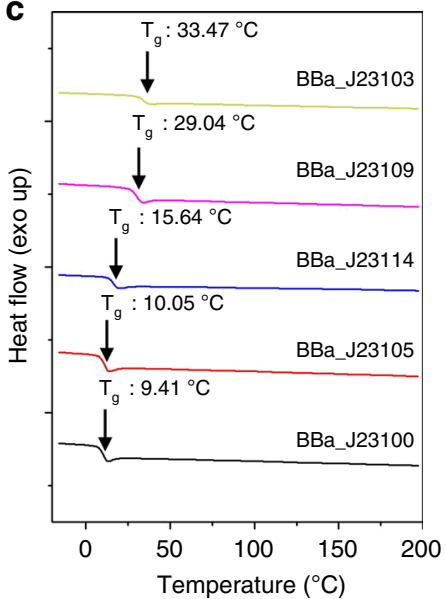
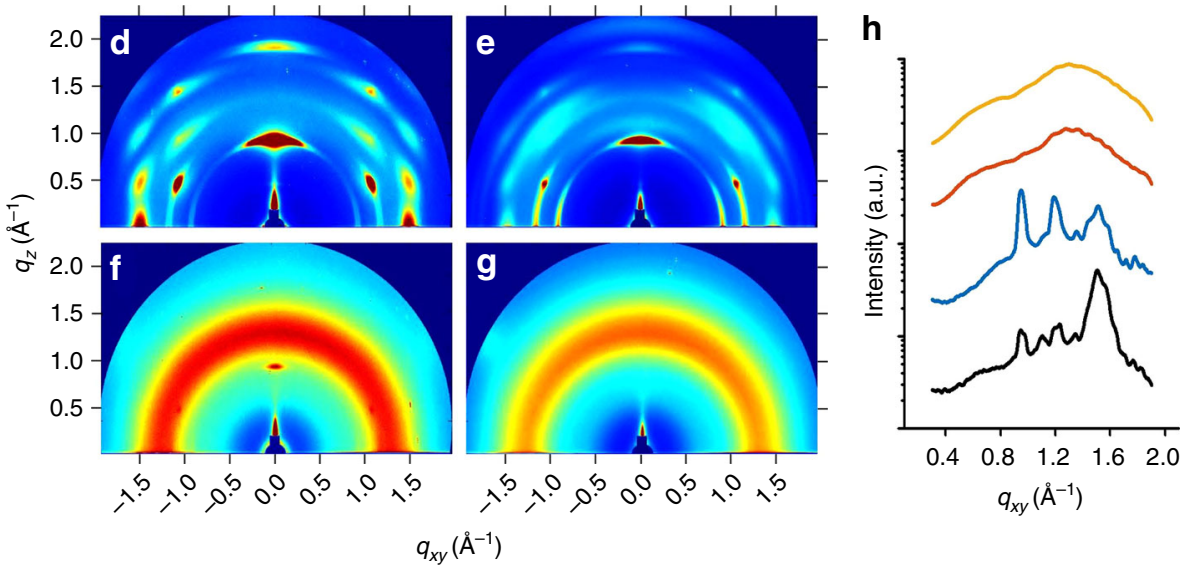

i

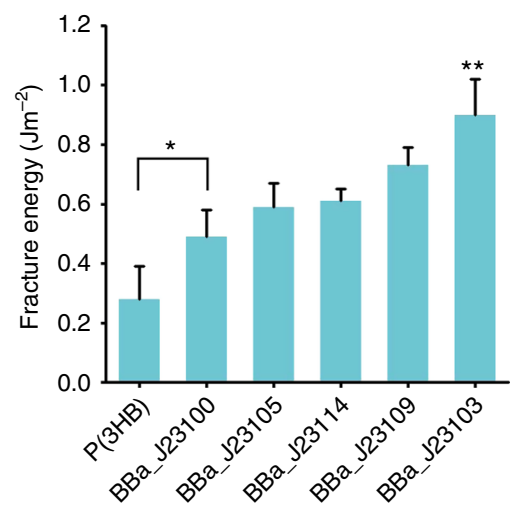

Fig. 4 Characterization of synthetic promoter driven aromatic polyesters. a Scheme of the PhaAB expression cassette. Analysis of D-phenyllactate monomer fraction $\mathbf{b}$ and differential scanning calorimetry (DSC) analysis $\mathbf{c}$ of copolymers produced by XB201TBAL strain expressing PhaAB under the synthetic Anderson promoters (BBa_J23100, BBa_J23105, BBa_J23114, BBa_J23109, and BBa_J23103) followed by overexpression of AroG ${ }^{\mathrm{fbr}}$, PheA ${ }^{\mathrm{fbr}}$, FldH, HadA, and PhaC1437. d-g Grazing-incidence X-ray scattering (GIXS) patterns of poly(3HB) d, poly(16.8 mol\% D-lactate-co-80.8 mol\% 3HB-co-1.6 mol\% Dphenyllactate-co-0.8 mol\% D-4-hydroxyphenyllactate) e, poly( $80.8 \mathrm{~mol} \% 3 \mathrm{HB}-\mathrm{co}-35.5 \mathrm{~mol} \% \mathrm{D}$-phenyllactate) f, and poly(52.1 mol\% $3 \mathrm{HB}-c 0-47.9 \mathrm{~mol} \% \mathrm{D}$ phenyllactate) $\mathbf{g}$. Colors indicate the intensity counts ranging from 0 (blue) to $10^{5}$ (red). $\mathbf{h}$ In-plane line cut of GIXS patterns of each polymer (d black; $\mathbf{e}$ blue; $\mathbf{f}$ orange; $\mathbf{g}$ yellow). i Dual cantilever beam (DCB) test of polymers. ${ }^{\star} P<0.05$ and ${ }^{\star \star} P<0.01$, by two-tailed $t$-test. Error bars represent s.d. of $n=3$ technical replicates

3HB-co-18.0 mol\% D-phenyllactate-co-48.7 mol\% D-3-hydroxy-3phenylpropionate) to the polymer content of $14.7 \mathrm{wt} \%$ of dry cell weight in MR medium containing $20 \mathrm{gl}^{-1}$ of glucose and $1 \mathrm{gl}^{-1}$ of sodium $3 \mathrm{HB}$ together with $0.5 \mathrm{gl}^{-1}$ of their corresponding monomers, respectively (Supplementary Fig. 10). These results strongly suggest that the engineered E. coli system expressing HadA and evolved PHA synthase can be broadly employed for the production of diverse aromatic polyesters.

Synthetic promoter based flux modulation. In the above studies, $3 \mathrm{HB}$ was supplemented to boost polymer accumulation as described earlier. Next, production of aromatic PHAs from glucose without 3HB supplementation was pursued in XB201TBAL strain by additionally expressing Ralstonia eutropha $\beta$ ketothiolase $(\mathrm{PhaA})$ and acetoacetyl-CoA reductase (PhaB). As expected, XB201TBAL strain expressing AroG ${ }^{\mathrm{fbr}}$, PheA ${ }^{\mathrm{fbr}}$, FldH, HadA, and PhaCl437 was able to produce poly $(86.2 \mathrm{~mol} \% 3 \mathrm{HB}-$ co-13.8 mol\% D-phenyllactate) to the polymer content of $18.0 \mathrm{wt}$ $\%$ of dry cell weight in MR medium containing $20 \mathrm{~g} \mathrm{l}^{-1}$ of glucose as a sole carbon source. In addition, production of aromatic PHAs having different monomer fractions, which is important for industrial applications, was also attempted by modulating metabolic fluxes of PhaAB using synthetic Anderson promoters (http://parts.igem.org/). Five different plasmids expressing PhaAB under five promoters of different strength were constructed and introduced into the XB201TBAL strain expressing AroG $^{\mathrm{fbr}}$, $\mathrm{PheA}^{\mathrm{fbr}}$, FldH, HadA, and PhaC1437. The D-phenyllactate monomer fraction could be modulated, showing increased fraction with decreased PhaAB expression; polymers having 11.0, $15.8,20.0,70.8$, and $84.5 \mathrm{~mol} \%$ of D-phenyllactate could be produced (Fig. 4a, b and Supplementary Table 2); it should be noted that by expressing PhaAB under BBa_J23103 promoter, a polymer having a very high D-phenyllactate fraction (poly $(15.5 \mathrm{~mol} \%$ $3 \mathrm{HB}-c o-84.5 \mathrm{~mol} \%$ D-phenyllactate)) could be produced to the polymer content of $4.3 \mathrm{wt} \%$ of dry cell weight (Fig. 4b). These results suggest that aromatic polyesters having different aromatic monomer fractions can be produced by modulating metabolic fluxes.

Next, the pH-stat fed-batch culture of E. coli XB201TBAL strain expressing AroG ${ }^{\mathrm{fbr}}$, PheA $\mathrm{A}^{\mathrm{fbr}}$, FldH, HadA, PhaC1437, and PhaAB under BBa_J23114 promoter was performed in a medium containing glucose without $3 \mathrm{HB}$ feeding. In $96 \mathrm{~h}, 2.5 \mathrm{gl}^{-1}$ of poly ( $67.6 \mathrm{~mol} \% 3 \mathrm{HB}-c o-32.4 \mathrm{~mol} \%$ D-phenyllactate) was produced with a polymer content of $43.8 \mathrm{wt} \%$, which demonstrated that aromatic polyester can be produced by one-step fermentation of 
engineered E. coli from glucose (Supplementary Fig. 9). To further enhance production of aromatic PHAs, gene expression system was optimized by replacing the $l d h A$ gene with the $f l d H$ gene in the chromosome of $E$. coli XB201TBA. In addition, the native promoter of the $l d h A$ gene was replaced with the strong trc promoter to increase $f l d H$ expression. Furthermore, we employed pulsed-feeding method (see Methods). The resulting E. coli XB201TBAF strain expressing AroG ${ }^{\mathrm{fbr}}$, PheA ${ }^{\mathrm{fbr}}$, HadA, PhaC1437, and PhaAB under BBa_J23114 promoter allowed production of $13.9 \mathrm{~g} \mathrm{l}^{-1}$ of poly(61.9 mol\% 3HB-co-38.1 mol\% Dphenyllactate) with a polymer content of $55.0 \mathrm{wt} \%$ from glucose by fed-batch fermentation (Supplementary Fig. 9). This titer $\left(13.9 \mathrm{gl}^{-1}\right)$ obtained is 5.56 -fold higher than that $\left(2.5 \mathrm{gl}^{-1}\right)$ obtained with the E. coli XB201TBAL strain expressing AroG ${ }^{\text {fbr }}$, $\mathrm{PheA}^{\mathrm{fbr}}$, FldH, HadA, PhaC1437, and PhaAB under BBa_J23114 promoter and is also much higher than that $\left(<1 \mathrm{gl}^{-1}\right)$ obtained by fed-batch culture of the E. coli XB201TBAL strain expressing AroG ${ }^{\mathrm{fbr}}$, PheA $\mathrm{A}^{\mathrm{fbr}}$, FldH, HadA, and PhaC1437 in a medium supplemented with glucose and sodium 3HB. These results demonstrate that aromatic PHAs could be successfully produced to a reasonably high concentration by fed-batch culture of the engineered strain (XB201TBAF strain expressing AroG $^{\mathrm{fbr}}$, $\mathrm{PheA}^{\mathrm{fbr}}$, HadA, PhaC1437, and PhaAB under BBa_J23114 promoter). Although we provided proof-of-concept fermentation results here, it is expected that further optimization of cultivation condition will allow more efficient production of aromatic polyesters by one-step fermentation from glucose.

Next, material properties of aromatic polyesters produced in this study were examined to see whether they are suitable for industrial applications. In the case of PET, the most widely used petroleum-derived aromatic polyester, fiber-grade, and bottlegrade PET have a number-average molecular weight $\left(M_{\mathrm{n}}\right.$; molecular mass) of $15-20 \mathrm{kDa}$ and $24-36 \mathrm{kDa}$, respectively. The $M_{\mathrm{n}}$ 's of aromatic polyesters produced in this study are also in the range of 3.6-24.9 kDa (Supplementary Table 2), showing a good potential to replace petroleum-based aromatic polymers. To examine the material properties of the aromatic polyesters produced by metabolically engineered E. coli strains (Fig. 4c-i and Supplementary Note 3), films of five different aromatic polyesters produced above and poly $(3 \mathrm{HB})$ as a control were manufactured. The critical cohesive fracture energy $\left(G_{c}\right)$ of poly (3HB) film was measured to be $0.28 \pm 0.11$ (SD) $\mathrm{J} \mathrm{m}^{-2}$, whereas the $G_{\mathrm{c}}$ increased as the aromatic monomer fraction increased from 11.0 to $84.5 \mathrm{~mol} \%$. The polymer film made of poly $(15.5 \mathrm{~mol}$ $\% 3 \mathrm{HB}-\mathrm{co}-84.5 \mathrm{~mol} \% \mathrm{D}$-phenyllactate), the polymer having the highest D-phenyllactate fraction, showed increased $G_{\mathrm{c}}$ of $0.90 \pm$ 0.12 (SD) $\mathrm{J} \mathrm{m}^{-2}$, which is mainly attributed to the reduced crystallinity and high glass transition temperature $\left(T_{\mathrm{g}}\right)$ of the polymer. These results suggest that aromatic polyesters produced by one-step direct fermentation of engineered E. coli strains can be used to replace those aromatic polyesters currently produced from petroleum.

\section{Discussion}

In this study, we report the development of a bacterial platform system that allows production of various aromatic polyesters by one-step fermentation. The key strategies for the successful fermentative production of aromatic polyesters from glucose include identification and use of a novel broad substrate range CoAtransferase for activating aromatic compounds to their CoA derivatives and a mutant PHA synthase capable of polymerizing these aromatic CoA derivatives, and design and optimization of metabolic pathways to overproduce corresponding aromatic monomers in vivo. As demonstrated for several aromatic monomers, this system has a potential to be used for the production of even more diverse aromatic polymers. For example, HadA (or related enzymes) and PHA synthase can be further engineered to accept desired aromatic monomers of interest. Recently, the crystal structure of $R$. eutropha PHA synthase has been determined ${ }^{24,25}$. This structure can serve as a model for rational protein engineering of various PHA synthases to broaden the substrate utilization range. It is expected that the bacterial platform system developed here will help establish sustainable bioprocesses for the production of aromatic polyesters from renewable non-food biomass to substitute petroleum-based counterparts.

\section{Methods}

Plasmids and construction of bacterial strains. All chemicals used in this study were purchased from Sigma-Aldrich unless noted otherwise. All bacterial strains and plasmids used in this study were listed in Supplementary Table 3. E. coli XL1Blue (Stratagene Cloning Systems, La Jolla, CA) was used for general gene cloning and all DNA manipulations were performed following the standard procedures ${ }^{26}$. PCR was performed with the C1000 Thermal Cycler (Bio-Rad, Hercules, CA).

Primers used in this study (Supplementary Table 4) were synthesized by Genotech (Daejeon, Korea).

The pPs619C1437Pct540 was used for the expression of the Pseudomonas sp. 6-19 PHA synthase gene (phaC; ACM68707.1) containing quadruple mutations of E130D, S325T, S477G, and Q481K (PhaC1437), and the C. propionicum Pct gene containing mutation of V193A and four silent nucleotide mutations of T78C, T669C, A1125G, and T1158C (Pct540) ${ }^{10}$

To construct the pET22b-hisPCT540, the pct540 gene was amplified by PCR using the primers Pcthis-F and (His) ${ }_{6}$-tagged Pcthis- $\mathrm{R}$ using pPs619C1437Pct540 as template. The amplified DNA fragment was digested with NdeI and Bam HI and ligated with NdeI-BamHI-digested pET22b(+) plasmid. To construct pET22b his4CL, the $S$. coelicolor 4-coumarate:CoA ligase gene $(4 C L)$ was amplified with the primers 4 CLhis-F and 4CLhis-R using the genomic DNA of $S$. coelicolor as a template. The PCR product was digested with EcoRI and Sbfl, and ligated with EcoRI-Sbfl-digested pET22b(+). To construct pET22b-his4CL(A294G), the first 885 bp DNA fragment was amplified with the primers 4CLhis-F and 4CLmut-R containing a single mutated base (G881C). The second 720 bp DNA fragment was amplified with the primers 4CLmut-F containing a single mutated base (C881G) and 4 CLhis-R. Then the complete $1,587 \mathrm{bp}$ DNA fragment was amplified with the primers 4CLhis-F and 4CLhis-R by overlapping PCR using the mixed two fragments as a template. To make pET22b-hisFldA, the C. botulinum A str. ATCC 3502 cinnamoyl-CoA:phenyllactate CoA-transferase gene $(\mathrm{fldA})$ was used. For better expression of the fldA gene in recombinant $E$. coli, its codon usage was further optimized to E. coli (synthesized at GenScript, Piscataway, NJ, and cloned into a vector to make plasmid pUC57-FldAopt) and E. coli codon-optimized fldA gene was amplified with the primers FldAhis-F and FldAhis-R using pUC57FldAopt as a template. The PCR product was digested with NdeI and HindIII, and ligated with NdeI-HindIII-digested pET22b $(+)$ plasmid.

To construct pKM212-AroG ${ }^{\text {fbr }}$ for the expression of the feedback inhibition resistant, DAHP synthase gene (aroG) mutant was amplified by PCR with the primers AroG-F and AroG-R using the plasmid pTyr-a ${ }^{27}$ as a template. The PCR product was digested with EcoRI and HindIII, and ligated with EcoRI-HindIIIdigested pKM212-MCS ${ }^{12}$. The pKM212-AroG ${ }^{\mathrm{fbr}} \mathrm{PheA}^{\mathrm{fbr}}$ plasmid was constructed as follows. First, the 991 bp DNA fragment was amplified by PCR from the genomic DNA of $E$. coli using primers PheA-F and PheAmut-R, which contains a single mutated base (T976G). Second, 200 bp DNA fragment was amplified from the genomic DNA of $E$. coli using the primers PheAmut-F containing a single mutated base (A976C) and PheA-R. Then, complete 1,161 bp DNA fragment of the $p h e A^{f b r}$ gene was amplified with the primers PheA-F and PheA-R by overlapping PCR using mixed two fragments as a template. The PCR product was digested with HindIII and ligated with HindIII-digested pKM212-AroG ${ }^{\text {fbr }}$. To construct pKM212-AroG ${ }^{\mathrm{fr}} \mathrm{PAL}$, the codon usage of Streptomyces maritimus PAL gene was further optimized to E. coli (synthesized at GenScript, and cloned into a vector to make plasmid pUC57-PALopt). The E. coli codon-optimized PAL was amplified by PCR with the primers PAL-Hin-F and PAL-Hin-R using pUC57-PALopt. The PCR product was digested with HindIII and ligated with HindIII-digested pKM212AroG $^{\mathrm{fbr}}$. For the construction of pACYC-FldH, the C. botulinum A str. ATCC 3502 D-phenyllactate dehydrogenase gene $(f l d H)$ was used. The codon usage of the $f l d H$ gene was optimized to E. coli (synthesized at GenScript and cloned into a vector to make plasmid pUC57-FldHopt) and the E. coli codon optimized fldH gene was amplified with the primers FldH-F and FldH-R using the pUC57-FldHopt as a template. The PCR product was digested with BamHI and HindIII, and ligated with BamHI-HindIII-digested pTrc99A to make pTrc-FldH. Then, the $\mathrm{fldH}$ gene attached to the $t r c$ promoter and $r r n B$ terminator was amplified by PCR with the primers Trc-F and Ter-R using pTrc-FldH as a template. The PCR product was digested with XhoI and SacI, and ligated with XhoI-SacI-digested pACYC184KS to construct pACYC-FldH.

To construct pACYC-4CL(A294G), the mutant 4CL having amino acid change (A294G) was amplified by PCR with the primers Trc-F and Ter-R using the 
plasmid pET22b-his4CL(A294G) as a template. The PCR product was digested with $\mathrm{XhoI}$ and SacI, and ligated with XhoI-SacI-digested pACYC184KS to construct pACYC-4CL(A294G). pACYC-4CL(A294G)FldA was constructed by cloning the fldA gene amplified by PCR using the primers FldA-F and FldA-R containing C-terminal (His) ${ }_{6}$-tag from pET22b-hisFldA as a template into pACYC4CL(A294G) at Sbfl site. To make pACYC-4CL(A294G)FldAH, the fldH gene was amplified by PCR with the primers FldH-sbF and FldH-hiR using the plasmid pACYC-FldH as a template. The PCR product was digested with SbfI and HindIII sites, and ligated with SbfI-HindIII-digested pACYC-4CL(A294G)FldA to construct pACYC-4CL(A294G)FldAH.

To construct pET22b-hisHadA, the C. difficile HadA gene (hadA; AAV40822.1) fused with C-terminal (His) ${ }_{6}$-tag was amplified by PCR using the primers HadAhisF and HadA-hisR using the genomic DNA of C. difficile as a template. The PCR product was digested with NdeI and NotI, and ligated with NdeI-NotI-digested pET22b (+) plasmid. pPs619C1437-HadA was constructed by replacing the pct540 gene in pPs619C1437Pct540 with the C. difficile hadA gene that was amplified by PCR with primers HadA-sbF and HadA-ndR using the genomic DNA of C. difficile as a template. The PCR product was digested with SbfI and NdeI, and ligated with Sbfi-NdeI-digested DNA fragment of pPs619C1437Pct540.

For the construction of pKM212-GPE_PhaAB, the R. eutropha PHA biosynthesis operon promoter, the $\beta$-ketothiolase (phaA), and acetoacetyl-CoA reductase $(p h a B)$ genes were amplified by PCR with the primers PhaAB-BamF and $\mathrm{PhaAB}$-sbR using plasmid $\mathrm{pCnAB}{ }^{7}$ as a template. The PCR product was digested with BamHI and Sbfl, and ligated with BamHI-Sbfl-digested pKM212-

AroG ${ }^{\mathrm{fbr} P h e} \mathrm{~A}^{\mathrm{fbr}}$. To construct plasmids having different expression level of the phaAB genes under synthetic Anderson promoters (http://parts.igem.org/), the phaAB operon linked with BBa_J23100 was amplified with primer 100-Kpn-F and PhaB-Bam-R using the genomic DNA of $R$. eutropha as a template. The PCR product was digested with $K p n I$ and $B a m H I$, and ligated with KpnI-BamHIdigested pKM212-AroG ${ }^{\mathrm{fbr}} \mathrm{PheA}^{\mathrm{fbr}}$. Then, BBa_J23100 region was replaced with $\mathrm{BBa} \_J 23105, \mathrm{BBa} \_J 23114, \mathrm{BBa} \_J 23109$, and BBa_J23103 promoters, having relative promoter strengths of $1,0.24,0.10,0.04$, and 0.01 , respectively (http://parts.igem. $\mathrm{org} /$ ) through the same manner using corresponding primers and restriction enzymes as mentioned above. Deletion of genes in the chromosome of E. coli XL1Blue was performed by the one step inactivation method ${ }^{28}$. The primers used are listed in Supplementary Table 4. For replacements of $l d h A$ gene and its native promoter with the $f l d H$ gene and strong trc promoter in the chromosome of $E$. coli XB201TBA, plasmid pMtrcFldH, bearing a trc promoter and $f l d H$ gene downstream of the lox66-cat-lox71 cassette, was used.

In silico flux response analysis. The genome-scale metabolic model of $E$. coli iJO1366 ${ }^{29}$, comprising 2,251 metabolic reactions and 1,135 metabolites, was used for in silico flux response analysis to examine the effects of central and aromatic amino acid biosynthesis reactions on the D-phenyllactic acid production ${ }^{30}$. In order to reflect the genotype of XB201T strain in the iJO1366, heterologous metabolic reactions biosynthesizing D-phenyllactate ( $f l d H$ gene) were additionally introduced to the model and, and flux values of native $E$. coli reactions were constrained to zero for the genes knocked out in the XB201T strain. For the implementation of in silico flux response analysis, the flux values of each central and aromatic amino acid biosynthesis reaction were constrained from their minimum values to the maximum values. D-phenyllactic acid production rate (D-phenyllactate dehydrogenase) was subsequently maximized as an objective function at each constraint point where flux values of each central and aromatic amino acid biosynthesis reaction were fixed. The relationships between D-phenyllactic acid production rate and fluxes of each central and aromatic amino acid biosynthesis reaction were categorized into six types based on the flux patterns obtained from the in silico flux response analysis. Reactions that were predicted to be negatively correlated with Dphenyllactic acid production rate were identified as potential knockout candidates. During the simulation, the glucose uptake rate was set at $10 \mathrm{mmol}$ per gram of dry cell weight per hour. All simulations were performed in Python environment with Gurobi Optimizer 6.0 and GurobiPy package (Gurobi Optimization, Inc., Houston, TX). Reading, writing, and manipulation of the COBRA-compliant SBML files were implemented using COBRApy ${ }^{31}$

Culture condition. Luria-Bertani (LB) medium (containing per liter: $10 \mathrm{~g}$ tryptone, $5 \mathrm{~g}$ yeast extract and $10 \mathrm{~g} \mathrm{NaCl}$ ) was used for cultivation of E. coli for DNA manipulations. Recombinant $E$. coli XL1-Blue strains were cultured in a chemically defined MR medium at $30^{\circ} \mathrm{C}$ in a rotary shaker at 200 r.p.m. for the production of PHAs. MR medium (pH 7.0) contains (per liter): $6.67 \mathrm{~g} \mathrm{KH}_{2} \mathrm{PO}_{4}, 4 \mathrm{~g}\left(\mathrm{NH}_{4}\right)_{2} \mathrm{HPO}_{4}$, $0.8 \mathrm{~g} \mathrm{MgSO}_{4} \cdot 7 \mathrm{H}_{2} \mathrm{O}, 0.8 \mathrm{~g}$ citric acid, and $5 \mathrm{ml}$ trace metal solution. The trace metal solution contains (per liter of $0.5 \mathrm{M} \mathrm{HCl}$ ): $10 \mathrm{~g} \mathrm{FeSO}_{4} \cdot 7 \mathrm{H}_{2} \mathrm{O}, 2 \mathrm{~g} \mathrm{CaCl}_{2}, 2.2 \mathrm{~g}$ $\mathrm{ZnSO}_{4} \cdot 7 \mathrm{H}_{2} \mathrm{O}, 0.5 \mathrm{~g} \mathrm{MnSO}_{4} \cdot 4 \mathrm{H}_{2} \mathrm{O}, 1 \mathrm{~g} \mathrm{CuSO}_{4} \cdot 5 \mathrm{H}_{2} \mathrm{O}, 0.1 \mathrm{~g}\left(\mathrm{NH}_{4}\right)_{6} \mathrm{Mo}_{7} \mathrm{O}_{24} \cdot 4 \mathrm{H}_{2} \mathrm{O}$, and $0.02 \mathrm{~g} \mathrm{Na}_{2} \mathrm{~B}_{4} \mathrm{O}_{7} \cdot 10 \mathrm{H}_{2} \mathrm{O}$. Glucose, $\mathrm{MgSO}_{4} \cdot 7 \mathrm{H}_{2} \mathrm{O}$, sodium $3 \mathrm{HB}$ (Acros Organics), D-phenyllactic acid, D-mandelic acid, and D-3-hydroxy-3-phenylpropionic acid were sterilized separately. When the $\operatorname{tyr} B$ and $a s p C$ deletion strains were cultivated, $0.2 \mathrm{~g} \mathrm{l}^{-1}$ of L-tyrosine, $0.2 \mathrm{~g} \mathrm{l}^{-1}$ of L-phenylalanine, and $3 \mathrm{~g} \mathrm{l}^{-1}$ of L-aspartic acid were added to the culture medium. Sodium $3 \mathrm{HB}\left(1 \mathrm{~g} \mathrm{l}^{-1}\right)$ was added to the culture medium when it was needed. Ampicillin $\left(\mathrm{Ap}, 50 \mu \mathrm{g} \mathrm{m}^{-1}\right)$, kanamycin $(\mathrm{Km}, 30 \mu \mathrm{g}$ $\left.\mathrm{ml}^{-1}\right)$, and chloramphenicol $\left(\mathrm{Cm}, 34 \mu \mathrm{g} \mathrm{ml}^{-1}\right)$ were added to the medium according to the resistant marker of employed plasmids.
Fed-batch cultures were carried out in a 6.61 Bioreactor (Bioflow 3000; New Brunswick Scientific Co., Edison, NJ) containing 21 of MR medium supplemented with $20 \mathrm{gl}^{-1}$ of glucose and $1 \mathrm{gl}^{-1}$ of sodium $3 \mathrm{HB}$. The dissolved oxygen concentration was maintained above $40 \%$ of air saturation by automatically supplying air with the rate of $21 \mathrm{~min}^{-1}$ and by automatically controlling the agitation speed from 200 to 1,000 r.p.m. The culture $\mathrm{pH}$ was controlled at 7.0 by $28 \%(\mathrm{v} / \mathrm{v})$ ammonia solution. The feeding solution contains (per liter): $700 \mathrm{~g}$ glucose, $15 \mathrm{~g} \mathrm{MgSO}_{4} \cdot 7 \mathrm{H}_{2} \mathrm{O}$, and $250 \mathrm{mg}$ thiamine. The fed-batch fermentation was performed by $\mathrm{pH}$-stat strategy and also by pulsed-feeding strategy. For the $\mathrm{pH}$-stat fed-batch cultures, the $\mathrm{pH}$ of fermentation broth was maintained at $\mathrm{pH} 7.0$ and the feeding solution was automatically fed when the $\mathrm{pH}$ rose above 7.02 as a set point. For pulsed-feeding strategy, feeding solution was added when the glucose concentration in the fermentation broth fell below $\mathrm{g} \mathrm{g}^{-1}$ to increase the glucose concentration in the culture broth to 20 (in the case of 3HB supplemented fedbatch cultures) or $10 \mathrm{gl}^{-1}$ (without 3HB supplemented fed-batch cultures).

Pct and HadA enzyme assay. To assay the activity of Pct540 and HadA, E. coli BL21 (DE3) harboring pET22b-hisPct540 or pET22b-hisHadA was cultured in LB medium at $30^{\circ} \mathrm{C}$ and each enzyme was purified by using Talon metal affinity resin (Clontech, Mountain View, CA). The activities of Pct540 and HadA were measured as follows: the reaction was carried out for $30 \mathrm{~min}$ at $30^{\circ} \mathrm{C}$ in $50 \mathrm{mM}$ phosphate buffer ( $\mathrm{pH}$ 7.5) containing $0.1 \mathrm{mM}$ acetyl-CoA and $10 \mathrm{mM}$ substrates by adding 15 $\mu \mathrm{g}$ of purified enzyme. After the above reaction, $1 \mathrm{mM}$ oxaloacetate, $5 \mu \mathrm{g}$ of citrate

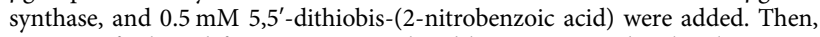
amount of released free $\mathrm{CoA}$ was analysed by measuring the absorbance at 412 $\mathrm{nm}^{32}$. One unit of enzyme activity was defined as the formation of $1 \mu \mathrm{mol}$ of corresponding CoA derivative from the substrate per minute. The specific activity was described as unit $\mathrm{mg}^{-1}$ protein.

4CL enzyme assay. The activity of $4 \mathrm{CL}$ was determined by the spectrophotometric assay as follows: the changes in absorbance during cinnamoyl-CoA formation were monitored at wavelengths of $311 \mathrm{~nm}$. The reaction mixture contained $400 \mathrm{mM}$ Tris- $\mathrm{HCl}$ ( $\mathrm{pH}$ 7.8), $5 \mathrm{mM}$ ATP, $5 \mathrm{mM} \mathrm{MgCl}$, $0.3 \mathrm{mM} \mathrm{CoA}, 0.5$ $\mathrm{mM}$ cinnamic acid, and $50 \mu \mathrm{g}$ of $4 \mathrm{CL}$ in total volume of $1 \mathrm{ml}$. The reaction was started by the adding $4 \mathrm{CL}$ enzyme at $30^{\circ} \mathrm{C}$ for $5 \mathrm{~min}$. The change in absorbance was measured by successive scanning of the wavelength with a spectrophotometer ${ }^{18,33}$

Cinnamoyl-CoA preparation and FIdA enzyme assay. The cinnamoyl-CoA for FldA reaction was prepared as follows: the OASIS HLB SPE cartridge (Waters, Milford, MA) was first conditioned with $3 \mathrm{ml}$ of methanol followed by addition of $3 \mathrm{ml}$ of $0.15 \%$ trichloroacetic acid (TCA) solution. The 4CL enzyme assay mixture was applied to the cartridge followed by addition of $2 \mathrm{ml}$ of $0.15 \%$ TCA solution. The attached CoA-thioesters were eluted with $1 \mathrm{ml}$ of methanol-ammonium hydroxide $(99: 1, \mathrm{v} / \mathrm{v})$ and then the sample was further evaporated to dryness at room temperature by vacuum centrifugation at $15,493 \times g$. The sample was dissolved with $50 \mathrm{mM}$ phosphate buffer (pH 7.5). The reaction was carried out for $1 \mathrm{~h}$ at $30^{\circ} \mathrm{C}$ in $50 \mathrm{mM}$ phosphate buffer ( $\mathrm{pH} 7.5$ ) containing cinnamoyl-CoA and 10 $\mathrm{mM}$ substrates by adding $10 \mu \mathrm{g}$ of purified FldA. After reaction, the consumption of cinnamoyl-CoA was measured by the spectrophotometric assay by measuring the absorbance at $311 \mathrm{~nm}$.

Analysis of aliphatic and aromatic acyl-CoAs. The formation of CoA-thioesters was confirmed by using high-performance liquid chromatography (HPLC)-mass spectrometry (MS). For the analysis of aliphatic and aromatic acyl-CoAs, samples were prepared by method described above in cinnamoyl-CoA preparation. The prepared samples were dissolved with $1 \mathrm{ml}$ of water for analysis and were subjected to HPLC (1100 series HPLC, Agilent Technologies, Palo Alto, CA) equipped with MS (LC/MSD VL, Agilent) and Eclipse XDB-C18 column $(5 \mu \mathrm{m}, 4.6 \times 150 \mathrm{~mm}$, Agilent).

Cell growth and metabolite analysis. Glucose concentration was measured using glucose analyser (model 2700 STAT, Yellow Spring Instrument, OH). Cell growth was monitored by measuring absorbance at $600 \mathrm{~nm}\left(\mathrm{OD}_{600}\right)$ using an Ultrospec 3000 spectrophotometer (Amersham Biosciences, Uppsala, Sweden). The metabolites (acetate, formate, lactate, succinate, and pyruvate) were analysed by HPLC (1515 isocratic HPLC pump, Waters) equipped with refractive index detector (2414, Waters) and MetaCarb $87 \mathrm{H}$ column (Agilent). Elution was performed with $0.01 \mathrm{~N} \mathrm{H}_{2} \mathrm{SO}_{4}$ at a flow rate of $0.5 \mathrm{ml} \mathrm{min}^{-1}$ at $25^{\circ} \mathrm{C}$. D-Phenyllactic acid, D-4hydroxyphenyllactic acid, L-tyrosine, and L-phenylalanine were analysed by HPLC (1100 series HPLC, Agilent Technologies) equipped with UV/VIS (G1315B, Agilent), refractive index (Shodex RI-71; Tokyo, Japan) detector and a ZorbaxSB-C18 column $(150 \mathrm{~mm} \times 4.6 \mathrm{~mm}, 5 \mu \mathrm{m}$, Agilent). Elution was performed with mobile phase A (Water- $0.1 \%$ trifluoroacetic acid) and mobile phase B (Acetonitrile). A linear gradient scheme started from $10 \%$ of mobile phase A and the fraction of mobile phase A was increased up to $50 \%$. The flow rate was $1 \mathrm{ml} \mathrm{min}^{-1}$ and analytes were detected at $220 \mathrm{~nm}$. 
Polymer analysis. The polymer contents and monomer compositions of synthesized PHAs were determined by gas chromatography (GC) or GC-MS (Supplementary Methods $)^{34}$. The collected cells were washed three times with distilled water and then lyophilized for $24 \mathrm{~h}$. The PHAs in lyophilized cells were converted into corresponding hydroxymethyl esters by acid-catalyzed methanolysis ${ }^{33}$. The resulting methyl esters were analysed by GC (Agilent $6890 \mathrm{~N}$, Agilent) equipped with Agilent 7683 automatic injector, flame ionization detector, and a fused silica capillary column (ATTM-Wax, $30 \mathrm{~m}$, ID $0.53 \mathrm{~mm}$, film thickness $1.20 \mu \mathrm{m}$, Alltech). Polymers were extracted from the cells by chloroform extraction method $^{35,36}$. Polymer content is defined as the weight percentage of polymer concentration to dry cell concentration (wt\% of dry cell weight). The structure, molecular weights, and thermal properties of the polymers were determined by nuclear magnetic resonance spectroscopy, gel permeation chromatography, and differential scanning calorimetry, respectively (Supplementary Methods) ${ }^{8,9}$.

Transmission electron microscopy analysis. To confirm intracellular aromatic PHA synthesis in recombinant $E$. coli, transmission electron microscopy (TEM) analysis was performed. After $72 \mathrm{~h}$ of cultivation, $5 \mathrm{ml}$ of cells were collected and washed twice with phosphate-buffered saline ( $\mathrm{pH}$ 7.0). Cells were fixed by adding $2.5 \%(\mathrm{v} / \mathrm{v})$ glutaraldehyde in phosphate-buffered saline $(\mathrm{pH} 7.0)$ and $1 \%(\mathrm{v} / \mathrm{v})$ osmium tetroxide. Then, the samples were dehydrated and embedded in Epon 812. The ultra-thin section was carried out by Ultra-Microtome (Leica Ultracut UCT, Leica, Austria). The section was stained with $2 \%$ uranyl acetate and lead citrate. TEM analysis was performed using Bio-TEM (Tecnai G2 Spirit, FEI, Hilsboro, OR) at the Korea Basic Science institute.

Grazing incidence $\mathbf{X}$-ray scattering measurements. The samples for grazing incidence X-ray scattering (GIXS) measurements were prepared by spin coating of copolymer solutions in chloroform (2 wt\%) on silicon substrate. GIXS measurements were performed on beamline 3C in the Pohang Accelerator Laboratory (South Korea). X-rays with energy of $11.075 \mathrm{keV}$ were used and the off-specular scattering was recorded using a MAR-CCD area detector and sample to detector distance was maintained to be $30 \mathrm{~cm}$. The incidence angle of the X-ray beam $\left(\sim 0.12^{\circ}\right)$ was used to allow for complete penetration of X-ray beam into the polymer film.

Dual cantilever beam test. The dual cantilever beam (DCB) specimens were prepared with the geometry of glass/aromatic PHAs/Au/epoxy/glass. A series of PHAs was dissolved in chloroform at the concentration of $2 \mathrm{wt} \%$ and then spincoated on a bare glass substrate. After sufficient drying of the residual chloroform in a vacuum oven, a $50 \mathrm{~nm}$-thick Au layer is deposited on the top of the polymer films by thermal evaporation. And then, the specimen was cut into $8 \mathrm{~mm} \times 25.4$ mm-long glass beam using Dicing Machine (DAD3350, DISCO Co., Japan) in order to prevent edge defects in the glass substrates. Finally, a dummy glass substrate coated by B-stage epoxy (Epo-Tek M10-D; Epoxy Technology) with a $1 \mu \mathrm{m}$ thickness was attached to complete the preparation of the DCB specimens. Two aluminum taps were attached at the specimen to apply loading and delaminate the DCB specimen. The cohesion energy was measured using a high-precision micromechanical test system (Delaminator Adhesion Test System, DTS Company, Menlo Park, CA). Multiple loading/crack-growth/unloading cycles were performed under a constant displacement rate of $0.5 \mu \mathrm{m} \mathrm{s}^{-1}$, whereas the applied load was continuously recorded as a function of the displacement. The cohesion energy $\left(G_{c}\right)$ was calculated as the critical value of the strain energy release rate $\mathrm{r}^{37,38}$.

Statistical analysis. All experiments were performed at least three times. Means were compared using a two-sided Student's $t$-test. $P<0.05$ was considered significant. The investigators were blinded to the group allocation by randomly selecting single colonies multiple times. No statistical methods were used to predetermine sample size.

Data availability. The data supporting the findings of this study are available from the authors upon request.

Received: 1 July 2017 Accepted: 1 December 2017

Published online: 08 January 2018

\section{References}

1. Muller, R. J., Kleeberg, I. \& Deckwer, W. D. Biodegradation of polyesters containing aromatic constituents. J. Biotechnol. 86, 87-95 (2001).

2. Lee, S. Y. Bacterial polyhydroxyalkanoates. Biotechnol. Bioeng. 49, 1-14 (1996).

3. Chen, G.-Q. \& Patel, M. K. Plastics derived from biological sources: Present and Future: A technical and environmental review. Chem. Rev. 112, 2082-2099 (2012).

4. Steinbüchel, A. \& Lütke-Eversloh, T. Metabolic engineering and pathway construction for biotechnological production of relevant polyhydroxyalkanoates in microorganisms. Biochem. Eng. J. 16, 81-96 (2003).
5. Steinbüchel, A. \& Valentin, H. E. Diversity of bacterial polyhydroxyalkanoic acids. FEMS Microbiol. Lett. 128, 219-228 (1995).

6. Witholt, B. \& Kessler, B. Perspectives of medium chain length poly (hydroxyalkanoates), a versatile set of bacterial bioplastics. Curr. Opin. Biotechnol. 10, 279-285 (1999).

7. Choi, S. Y. et al. One-step fermentative production of poly(lactate-co-glycolate) from carbohydrates in Escherichia coli. Nat. Biotechnol. 34, 435-440 (2016).

8. Taguchi, S. et al. A microbial factory for lactate-based polyesters using a lactatepolymerizing enzyme. Proc. Natl Acad. Sci. USA 105, 17323-17327 (2008).

9. Jung, Y. K., Kim, T. Y., Park, S. J. \& Lee, S. Y. Metabolic engineering of Escherichia coli for the production of polylactic acid and its copolymers. Biotechnol. Bioeng. 105, 161-171 (2010).

10. Yang, T. H. et al. Biosynthesis of polylactic acid and its copolymers using evolved propionate CoA transferase and PHA synthase. Biotechnol. Bioeng. 105, 150-160 (2010).

11. Jung, Y. K. \& Lee, S. Y. Efficient production of polylactic acid and its copolymers by metabolically engineered Escherichia coli. J. Biotechnol. 151, 94-101 (2011).

12. Park, S. J. et al. Metabolic engineering of Ralstonia eutropha for the biosynthesis of 2-hydroxyacid-containing polyhydroxyalkanoates. Metab. Eng. 20, 20-28 (2013).

13. Yang, J. E. et al. Biosynthesis of poly(2-hydroxyisovalerate-co-lactate) by metabolically engineered Escherichia coli. Biotechnol. J. 11, 1572-1585 (2016).

14. Fritzsche, K., Lenz, R. W. \& Fuller, R. C. An unusual bacterial polyester with a phenyl pendant group. Die Makromol. Chem. 191, 1957-1965 (1990).

15. Garcia, B. et al. Novel biodegradable aromatic plastics from a bacterial source Genetic and biochemical studies on a route of the phenylacetyl-coa catabolon. J. Biol. Chem. 274, 29228-29241 (1999).

16. Olivera, E. R. et al. Genetically engineered Pseudomonas: a factory of new bioplastics with broad applications. Environ. Microbiol. 3, 612-618 (2001).

17. Dickert, S., Pierik, A. J., Linder, D. \& Buckel, W. The involvement of coenzyme A esters in the dehydration of (R)-phenyllactate to (E)-cinnamate by Clostridium sporogenes. Eur. J. Biochem. 267, 3874-3884 (2000).

18. Kaneko, M., Ohnishi, Y. \& Horinouchi, S. Cinnamate:coenzyme A ligase from the filamentous bacterium streptomyces coelicolor A3(2). J. Bacteriol. 185, 20-27 (2003).

19. Dusha, I. \& Denes, G. Purification and properties of tyrosine-sensitive 3-deoxyD-arabino-heptolosonate-7-phosphate synthetase of Escherichia coli K12. Biochim. Biophys. Acta 438, 563-573 (1976).

20. Tribe, D. E., Camakaris, H. \& Pittard, J. Constitutive and repressivle enzymes of the common pathway of aromatic biosynthesis in Escherichia coli K-12: regulation of enzyme synthesis at different growth rates. J. Bacteriol. 127, 1085-1097 (1976).

21. Kikuchi, Y., Tsujimoto, K. \& Kurahashi, O. Mutational analysis of the feedback sites of phenylalanine-sensitive 3-deoxy-D-arabino-heptulosonate-7-phosphate synthase of Escherichia coli. Appl. Environ. Microbiol. 63, 761-762 (1997).

22. Zhou, H. Y., Liao, X. Y., Wang, T. W., Du, G. C. \& Chen, J. Enhanced Lphenylalanine biosynthesis by co-expression of $p h e A^{f b r}$ and $a r o F^{w t}$. Bioresour. Technol. 101, 4151-4156 (2010).

23. Kim, J., Darley, D. \& Buckel, W. 2-Hydroxyisocaproyl-CoA dehydratase and its activator from Clostridium difficile. Febs. J. 272, 550-561 (2005).

24. Kim, J., Kim, Y. J., Choi, S. Y., Lee, S. Y. \& Kim, K. J. Crystal structure of Ralstonia eutropha polyhydroxyalkanoate synthase C-terminal domain and reaction mechanisms. Biotechnol. J. 12, 1-12 (2016).

25. Kim, Y. J. et al. Structure and function of the N-terminal domain of Ralstonia eutropha polyhydroxyalkanoate synthase, and the proposed structure and mechanisms of the whole enzyme. Biotechnol. J. 12, 1-10 (2016).

26. Sambrook, J. \& Russell, D. W. Molecular Cloning: A Laboratory Manual 3rd edn (Cold Spring Harbor Laboratory Press, 2001).

27. Na, D. et al. Metabolic engineering of Escherichia coli using synthetic small regulatory RNAs. Nat. Biotechnol. 31, 170-174 (2013).

28. Datsenko, K. A. \& Wanner, B. L. One-step inactivation of chromosomal genes in Escherichia coli K-12 using PCR products. Proc. Natl Acad. Sci. USA 97, 6640-6645 (2000).

29. Orth, J. D. et al. A comprehensive genome-scale reconstruction of Escherichia coli metabolism-2011. Mol. Syst. Biol. 7, 535 (2011).

30. Lee, K. H., Park, J. H., Kim, T. Y., Kim, H. U. \& Lee, S. Y. Systems metabolic engineering of Escherichia coli for L-threonine production. Mol. Syst. Biol. 3, 149 (2007).

31. Ebrahim, A., Lerman, J. A., Palsson, B. O. \& Hyduke, D. R. COBRApy: constraintsbased reconstruction and analysis for Python. BMC Syst. Biol. 7, 74 (2013).

32. Lindenkamp, N., Schürmann, M. \& Steinbüchel, A. A propionate CoAtransferase of Ralstonia eutropha $\mathrm{H} 16$ with broad substrate specificity catalyzing the CoA thioester formation of various carboxylic acids. Appl. Microbiol. Biotechnol. 97, 7699-7709 (2013).

33. Knobloch, K. H. \& Hahlbrock, K. 4-Coumarate:CoA ligase from cell suspension cultures of Petroselinum hortense Hoffm. Partial purification, substrate specificity, and further properties. Arch. Biochem. Biophys. 184, 237-248 (1977). 
34. Braunegg, G., Sonnleitner, B. \& Lafferty, R. A rapid gas chromatographic method for the determination of poly- $\beta$-hydroxybutyric acid in microbial biomass. Appl. Microbiol. Biotechnol. 6, 29-37 (1978).

35. Choi, J. \& Lee, S. Y. Efficient and economical recovery of poly(3hydroxybutyrate) from recombinant Escherichia coli by simple digestion with chemicals. Biotechnol. Bioeng. 62, 546-553 (1999).

36. Jacquel, N., Lo, C. W., Wei, Y. H., Wu, H. S. \& Wang, S. S. Isolation and purification of bacterial poly (3-hydroxyalkanoates). Biochem. Eng. J. 39, 15-27 (2008).

37. Bruner, C., Miller, N. C., McGehee, M. D. \& Dauskardt, R. H. Molecular intercalation and cohesion of organic bulk heterojunction photovoltaic devices. Adv. Funct. Mater. 23, 2863-2871 (2013).

38. Lee, I., Kim, G. W., Yang, M. \& Kim, T. S. Simultaneously enhancing the cohesion and electrical conductivity of PEDOT: PSS conductive polymer films using DMSO additives. ACS Appl. Mater. Interfaces 8, 302-310 (2015).

\section{Acknowledgements}

This work was supported by the Intelligent Synthetic Biology Center through the Global Frontier Project (2011-0031963) and also by the Technology Development Program to Solve Climate Changes on Systems Metabolic Engineering for Biorefineries (NRF2012M1A2A2026556 and NRF-2012M1A2A2026557) from the Ministry of Science and ICT through the National Research Foundation of Korea.

\section{Author contributions}

S.Y.L. conceived the project. S.Y.L., J.E.Y., and S.J.P. generated ideas and designed research. J.E.Y. and S.J.P. performed research and analytical experiments. W.J.K. performed in silico metabolic simulation. J.E.Y., S.J.P., S.Y.L., H.J.K., B.J.K., J.S., and H.L. analysed data. J.E.Y., S.J.P., and S.Y.L. wrote the paper and all authors approved the final manuscript.

\section{Additional information}

Supplementary Information accompanies this paper at https://doi.org/10.1038/s41467017-02498-w.

Competing interests: S.Y.L., J.E.Y., and S.J.P. declare that they have competing financial interest as strains, enzymes, and genes described in this paper are patented and are of commercial interest. The remaining authors declare no competing financial interests.

Reprints and permission information is available online at http://npg.nature.com/ reprintsandpermissions/

Publisher's note: Springer Nature remains neutral with regard to jurisdictional claims in published maps and institutional affiliations.

(c) (i) Open Access This article is licensed under a Creative Commons Attribution 4.0 International License, which permits use, sharing, adaptation, distribution and reproduction in any medium or format, as long as you give appropriate credit to the original author(s) and the source, provide a link to the Creative Commons license, and indicate if changes were made. The images or other third party material in this article are included in the article's Creative Commons license, unless indicated otherwise in a credit line to the material. If material is not included in the article's Creative Commons license and your intended use is not permitted by statutory regulation or exceeds the permitted use, you will need to obtain permission directly from the copyright holder. To view a copy of this license, visit http://creativecommons.org/ licenses/by/4.0/.

(C) The Author(s) 2017 